\title{
Incidence of Chemotherapy-Induced
} Amenorrhea in Premenopausal Patients with Breast Cancer Following Adjuvant Chemotherapy with Anthracycline and Taxane

\author{
Nguyen Tien Quang, Pham Tuan Anh, Le Van Quang, Nguyen Van Hai, Nguyen Duc Huan, \\ Nguyen Cong Tin, Nguyen Thi Thanh Tam, Hoang Thu Hang, Nguyen Thi Thuy, \\ Do Thi Thanh Mai, Tran Hoang Nam, Le Thi Hang \\ Department of Optimal Clinical Care, National Cancer Hospital of Vietnam, Ha Noi, Vietnam \\ Email: phamtuananh@hmu.edu.vn
}

How to cite this paper: Quang, N.T., Anh, P.T., Van Quang, L., Van Hai, N., Huan, N.D., Tin, N.C., Tam, N.T.T., Hang, H.T., Thuy, N.T., Mai, D.T.T., Nam, T.H. and Hang, L.T. (2020) Incidence of Chemotherapy-Induced Amenorrhea in Premenopausal Patients with Breast Cancer Following Adjuvant Chemotherapy with Anthracycline and Taxane. Open Access Library Journal, 7: e5999.

https://doi.org/10.4236/oalib.1105999

Received: December 13, 2019

Accepted: January 5, 2020

Published: January 8, 2020

Copyright $\odot 2020$ by author(s) and Open Access Library Inc.

This work is licensed under the Creative Commons Attribution International License (CC BY 4.0).

http://creativecommons.org/licenses/by/4.0/

\begin{abstract}
Breast cancer (BC) is the second incidence and the fifth leading cause to cancer death in the worldwide. In developing countries, the number of $\mathrm{BC}$ cases is still increased annually accompanied with the changes of lifestyle and screening programs. Nevertheless, the majority of BC patients are diagnosed as non-curative stage. Thereby, systemic therapy always plays an important role in $\mathrm{BC}$ treatment strategies. Among chemotherapy regimens, the combination of anthracyclin and taxane as an adjuvant treatment has been proven the efficacy and safety in terms of prolonging survival. In this study, we evaluated the incidence of chemotherapy-induced amenorrhea in breast cancer patients who were treated with adjuvant anthracycline and taxane-based chemotherapy.
\end{abstract}

\section{Subject Areas \\ Oncology}

\section{Keywords}

Breast Cancer, Chemotherapy-Induced Amenorrhea, Adjuvant Anthracycline and Taxane-Based Chemotherapy

\section{Introduction}

Breast cancer (BC) is the most frequently diagnosed cancer in women of 
childbearing age. According to Globocan 2018, there are 15,229 new cases and 6,103 deaths from BC, the most common cancer in Vietnam. The incidence of BC is 26.4/100,000 people in 2018 [1].

Treatment BC is a combination of surgery, radiotherapy, chemotherapy (CT), endocrine, and biological therapy. The four cycles of anthracycline and cyclophosphamide, following by four cycles of taxane (4AC-4T) are considered to be the standard regimen for adjuvant treatment of BC, which has been shown to prolong the survival, but may cause early and prolong adverse effects such as amenorrhea leading to menopausal symptoms including hot flashes, sexual dysfunction, psychological stress and even osteoporosis. Although studies have shown that early menopause after CT is associated with better prognosis, early determination of menstruation is also associated with adverse health outcomes and poor quality of life. The risk of chemotherapy-induce amenorrhea ranges from $18 \%$ to $91 \%$ depending on the study [2] [3] [4]. In women over 40 years, this rate ranges from $61 \%$ to $97 \%$ [3]. This tremendous variation reflects that the incidence of chemotherapy-induce amenorrhea depends on variety of factors, such as age, patient characteristics, and even the definition of chemotherapy-induce amenorrhea. The most obvious factor was that women over the age of 40 had a higher risk of chemotherapy-induce amenorrhea than women under 40 years of age.

The adjuvant and neo-adjuvant regimen of $4 \mathrm{AC}-4 \mathrm{~T}$ is currently used in Vietnam, but no studies have evaluated the effects of this regimen on the amenorrhea in BC patients. Therefore, we conducted the study "Incidence of chemotherapy-induced amenorrhea in premenopausal patients with breast cancer following adjuvant chemotherapy with anthracycline and taxane”.

\section{Patients and Methods}

\subsection{Patients}

This is a retrospective study, enroll 111 patients with locoregional BC according to the tumor-lymph node-metastasis staging system classification. After surgery, these patients were treated with adjuvant CT by four cycles of doxorubicine and cyclophosphomide following by four cycles of paclitaxel at National Cancer Hospital of Viet Nam, from October 2015 to May 2018. The criteria of inclusions were breast cancer, premenopause, no history of surgical oophorectomy, no ovarian suppression or albation postchemotherapy, receive 8 cycles CT with cyclophosphamide, doxorubicin and paclitaxel. We sent questionnaires to 111 patients asking for information on their menopausal status. All of these patients answered these questions and were followed-up at least 12 months after finishing adjuvant CT. Amenorrhea was defined as the absence of menstruation during CT. Written informed consent was obtained from these patients for publication of their clinical date.

\subsection{Statistical Methods}

A Student $t$ test was used to assess whether there was an association between age, 
hormonal receptor status, and the presence of amenorrhea.

\section{Results}

\subsection{Patient Characteristics}

The median age at diagnosis was 41 years (range 22 - 51 years). All patients in our study were premenopausal at diagnosis. Most of patients were at stage II and had positive hormone receptor (Table 1 ).

\subsection{Incidence of Chemotherapy-Induced Amenorrhea}

One hundred eleven (100\%) of patients were completed with four cycles of anthracycline and cyclophosphamide followed by four cycles of taxane. All of these patients were followed-up at least 12 months after finished chemotherapy. After $8^{\text {th }}$ cycle, ratio of amenorrhea is the highest account for $82 \%$ (Table 2). The more cycles are performed, the higher ratio of amenorrhea is.

Out of 111 patients, 68 patients $(61.3 \%)$ had amenorrhea after the completition of chemotherapy (Figure 1). In our study, we analysed the ratio of amenorrhea every 3 months after finished chemotherapy. This ratio is decreased over the time.

Table 1. Patient characteristics.

\begin{tabular}{cc}
\hline Variables & No of patients (\%) \\
\hline Age (y), mean \pm SD & $41.4 \pm 5.9$ \\
Oral contraceptives: & $70(63.1)$ \\
Yes & $41(36.9)$ \\
No & \\
Staging: & $4(3.6)$ \\
I & $91(82)$ \\
II & $16(14.4)$ \\
III & \\
Histologic type: & $95(85.6)$ \\
Invasive ductal carcinoma & $8(7.2)$ \\
Invasive lobular carcinoma & $8(7.2)$ \\
Other & \\
Hormone receptor: & $70(63.1)$ \\
Positive & $41(36.9)$ \\
Negative &
\end{tabular}

Table 2. Incidence of amenorrhea during course treatment.

\begin{tabular}{cccccc}
\hline Cycles & Number $(\mathrm{n})$ & Rate $(\%)$ & Cycles & Number (n) & Rate (\%) \\
\hline 1 & 20 & 18.0 & 5 & 78 & 70.3 \\
2 & 35 & 31.5 & 6 & 85 & 76.6 \\
3 & 60 & 54.1 & 7 & 87 & 78.4 \\
4 & 74 & 66.7 & 8 & 91 & 82.0 \\
\hline
\end{tabular}




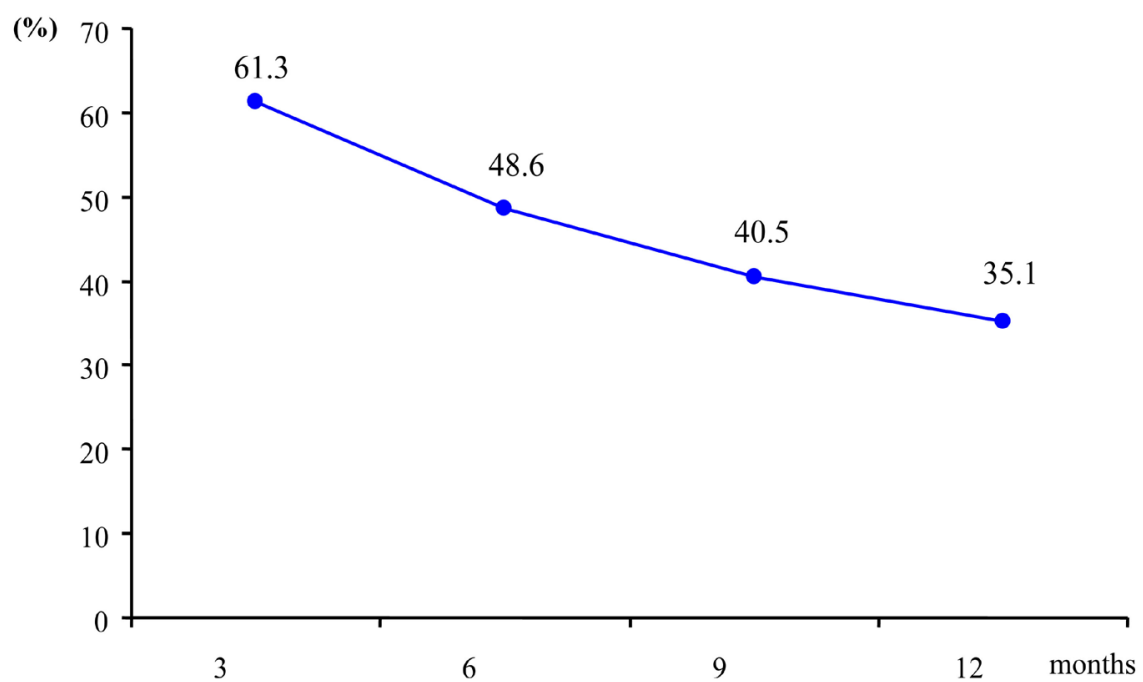

Figure 1. Ratio of amenorrhea after finished chemotherapy.

\subsection{Related Factors to the Incidence of Chemotherapy-Induced Amenorrhea}

We then analysed the association of age and hormonal status with the incidence of chemotherapy-induced amenorrhea. Age at diagnosis ( $\leq 40$ vs. $>40$ years) was significantly and independently associated with incidence of amenorrhea during CT $(\mathrm{p}=0.0008)$, but hormonal status was not significant (Table 3$)$.

\section{Discussion}

Of a total of 111 patients, the youngest patient was 22 years old and the oldest was 51 years, median age at diagnosis was $41.4 \pm 5.9$ years. Patients over 40 years old accounted for $50.5 \%$. This also reflects the fact that in our country, patients with breast cancer generally have a relatively young average age. Our findings are consistent with results from previous studies. In 1997, a study of 259 BC patients diagnosed from 1989 to 1992 showed that the median age was 45.2 years. According to Vu Hong Thang (1999), the 40 - 49-year-old age group was the most common, accounting for $47.8 \%$ [5]. An another study of 2158 BC patients showed the same result [6].

The median age of BC patients in our study was lower than other studies. In a study of Giuliano, the median age at diagnosis was 58 years [7], and was 59 years according to the study of Choi [8].

In our study, $66.7 \%$ of the patients were amenorrhetic after four cycles of AC. In addition to four cycles of paclitaxel, the incidence of chemotherapy-induced amenorrhea was $82.0 \%$. Our results are consistent with the results of Tham et al. In 2007, their study revealed that AC-T regimen produces amenorrhea more frequently compared to non taxane-based (e.g. AC) protocols, (61\% vs. 44\%) although this was not statistically significant [9]. Also, Martine et al found that 93\% of the cases the amenorrhea persisted beyond the duration of CT with FEC and $92.5 \%$ with $3 \mathrm{FEC} / 3 \mathrm{D}[10]$. 
Table 3. Related factors to the incidence of chemotherapy-induced amenorrhea.

\begin{tabular}{ccccccc}
\hline & & \multicolumn{2}{c}{ Amenorrhea } & \multicolumn{2}{c}{ No amenorrhea } & \multirow{2}{*}{ p value } \\
\cline { 3 - 6 } & & $\mathrm{N}$ & $\%$ & $\mathrm{~N}$ & $\%$ & \\
\hline \multirow{2}{*}{ Age } & 540 & 35 & 63.6 & 20 & 37.4 & \multirow{2}{*}{ Hormonal status } \\
& $>40$ & 56 & 100.0 & 0 & 0.0 & \\
& + & 55 & 78.6 & 15 & 21.4 & \multirow{2}{*}{0.222} \\
& - & 36 & 87.8 & 5 & 12.2 & \\
\hline
\end{tabular}

Of the 91 amenorrheic women, $38.7 \%$ had menses return at 3 months after $\mathrm{CT}$, and $64.9 \%$ at 12 months. Most of them were young, under 40 years olds. In a study of Martine, 36.5\% amenorrheic women had menses return at 6 months after CT by FEC [10]. In 2005, Fornier et al., studied in 166 BC patients under 40 years old. They were treated by sequential AC-T in cancer center Memorial Sloan-Kettering, New York. Among them, there was 141 (85\%) had menses return at 12 months after CT [2], higher than our study (57.1\%). The reason is that our study included patients under 40 years old.

In our study, we showed that the incidence of chemotherapy-induced amenorrhea associated with patient's age. The incidence of chemotherapy-induced amenorrhea in the patients' age over than 40 years was $100 \%$, significantly higher than the group under 40 years $(\mathrm{p}=0.0008)$. At months after adjuvant $\mathrm{CT}$ by CMF, Goldhirsch showed that the incidence of chemotherapy-induced amenorrhea was $33 \%$ in the group age lower 40 years old and $81 \%$ in the group under 40 years old [11].

\section{Conclusion}

In our study of $111 \mathrm{BC}$ patients treated with the adjuvant regimen of $4 \mathrm{AC}-4 \mathrm{~T}$, we have some conclusions: The mean age of the study's patient was $41.37 \pm 5.95$. After four cycles, the rate of amenorrhea is $66.7 \%$, after eight cycles is $82.0 \%$. At the time of six months, there is $48.6 \%$ and at twelve months is $35.1 \%$. The incidence of amenorrhea is closely related to age, and patients over 40 years of age have a significantly higher rate of amenorrhea than patients younger than 40 years of age.

\section{Conflicts of Interest}

The authors declare no conflicts of interest regarding the publication of this paper.

\section{References}

[1] https://gco.iarc.fr/today/online-analysis-multi-bars?v=2018\&mode=cancer\&mode population $=$ countries \&population $=900 \&$ populations $=900 \& \mathrm{key}=$ total $\& \mathrm{sex}=0 \& \mathrm{canc}$ er $=39 \&$ type $=0 \&$ statistic $=5 \&$ prevalence $=0$ \&population group $=0 \&$ ages group $\% 5 \mathrm{~B} \%$ $\underline{5 \mathrm{D}=0 \& \text { ages group } \% 5 \mathrm{~B} \% 5 \mathrm{D}=17 \& \mathrm{nb} \text { items }=10 \& \text { group cancer }=1 \text { \&include } \mathrm{nmsc}=1}$ \&include nmsc other $=1$ \&type multiple $=\% 257 \mathrm{~B} \% 2522$ inc $\% 2522 \% 253$ Atrue $\% 252 \mathrm{C}$ 
\%2522mort\%2522\%253Atrue\%252C\%2522prev\%2522\%253Afalse\%257D\&orientati on=horizontal\&type sort=1\&type nb items=\%257B $\% 2522$ top $\% 2522 \% 253$ Atrue $\% 2$

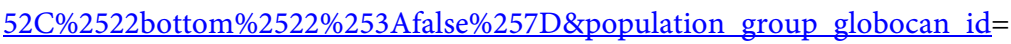

[2] Fornier, M.N., Modi, S., Panageas, K.S., Norton, L. and Hudis, C. (2005) Incidence of Chemotherapy-Induced, Long-Term Amenorrhea in Patients with Breast Carcinoma Age 40 Years and Younger after Adjuvant Anthracycline and Taxane. Cancer, 104, 1575-1579.

https://acsjournals.onlinelibrary.wiley.com/doi/full/10.1002/cncr.21385 https://doi.org/10.1002/cncr.21385

[3] Walshe, J.M., Denduluri, N. and Swain, S.M. (2006) Amenorrhea in Premenopausal Women after Adjuvant Chemotherapy for Breast Cancer. Journal of Clinical Oncology, 24, 5769-5779.

https://ascopubs.org/doi/abs/10.1200/JCO.2006.07.2793?journalCode=jco https://doi.org/10.1200//CO.2006.07.2793

[4] Jeon, S.J., Lee, J.I., Jeon, M.J. and Lee M. (2016) Prognostic Effects of Adjuvant Chemotherapy-Induced Amenorrhea and Subsequent Resumption of Menstruation for Premenopausal Breast Cancer Patients. Medicine, 95, e3301.

https://www.ncbi.nlm.nih.gov/pmc/articles/PMC4998816/ https://doi.org/10.1097/MD.0000000000003301

[5] Thang, V.H. (1999) Clinical and Pathology Cheresteristics of Stage I-II-III of Breast Cancer. Vietnam Journal of Oncology, 8, 54-57.

[6] Hung, N.C. and Thiep, T.V. (1999) Diagnosis and Treatment of Breast Cancer in Ho Chi Minh Oncology Hospital. Vietnam Journal of Oncology, 12, 197-206.

[7] Giuliano, A.E., Haigh, P.I., Brennan, M.B., Hansen, N.M., Kelley, M.C., Ye, W., Glass, E.C. and Turner, R.R. (2000) Prospective Observational Study of Sentinel Lymphadenectomy without Further Axillary Dissection in Patients with Sentinel Node-Negative Breast Cancer. Journal of Clinical Oncology, 18, 2553-2559. https://ascopubs.org/doi/10.1200/JCO.2000.18.13.2553 https://doi.org/10.1200/JCO.2000.18.13.2553

[8] Choi, S.H., Barsky, S.H. and Chang, H.R. (2003) Clinicopathologic Analysis of Sentinel Lymph Node Mapping in Early Breast Cancer. The Breast Journal, 9, 153-162. https://onlinelibrary.wiley.com/doi/abs/10.1046/j.1524-4741.2003.09304.x https://doi.org/10.1046/j.1524-4741.2003.09304.x

[9] Tham, Y.L., Sexton, K., Weiss, H., Elledge, R., Friedman, L.C. and Kramer, R. (2007) The Rates of Chemotherapy-Induced Amenorrhea in Patients Treated with Adjuvant Doxorubicin and Cyclophosphamide Followed by a Taxane. American Journal of Clinical Oncology, 30, 126-132.

https://insights.ovid.com/crossref?an=00000421-200704000-00004

https://doi.org/10.1097/01.coc.0000251398.57630.4f

[10] Berliere, M., Dalenc, F., Malingret, N., Vindevogel, A., Piette, P., Roche, H., Donnez, J., Symann, M, Kerger, J. and Machiels, P. (2008) Incidence of Reversible Amenorrhea in Women with Breast Cancer Undergoing Adjuvant Anthracycline-Based Chemotherapy with or without Docetaxel. BMC Cancer, 8, 56.

https://bmccancer.biomedcentral.com/articles/10.1186/1471-2407-8-56

https://doi.org/10.1186/1471-2407-8-56

[11] Goldhirsch, A., Gelber, R.D. and Castiglione, M. (1990) The Magnitude of Endocrine Effects of Adjuvant Chemotherapy for Premenopausal Breast Cancer Patients. Annals of Oncology, 1, 183-188.

https://academic.oup.com/annonc/article-abstract/1/3/183/146536?redirectedFrom =fulltext

https://doi.org/10.1093/oxfordjournals.annonc.a057718 